\title{
ACROCALLOSAL SYNDROME IN A 6-MONTH-OLD PAKISTANI INFANT
}

\author{
Rifayatullah Afridi ${ }^{1}$, Aneela Ambreen ${ }^{1}$, Faizan Ali Janjua ${ }^{1}$
}

1. Naseer Teaching Hospital

\begin{abstract}
Acrocallosal syndrome is a rare autosomal recessive disorder. The prevalence of the disease is not known but fewer than 55 cases have been published since 1979. ACS is characterized by the total or partial absence of the corpus callosum, Minor craniofacial anomalies (prominent forehead, hypertelorism, short nose with anteverted nostrils and large anterior fontanel) moderate to severe psychomotor retardation (with hypotonia), polydactyly or polysyndactyly. We are reporting a case of 6 month old infant child who was admitted to the hospital with pneumonia, measles, episodes of fits, large anterior fontanelle, prominent forehead, high arched palate, fused tongue, micrognathism, hypotonia, motor developmental delay, polysyndactyly and absent corpus callosum. His clinical diagnosis was confirmed by CT-Brain. The patient was managed for superimposed pneumonia and measles. He was provided with other supportive treatment as well as parental counseling, physiotherapy sessions, and multiple disciplinary approaches was undertaken for further management.
\end{abstract}

\section{KEY WORDS}

Acrocallosal syndrome, 6 month, Pakistani infant

\section{INTRODUCTION}

Acrocallosal syndrome (ACS) is an uncommon disorder, first described in 1979 by

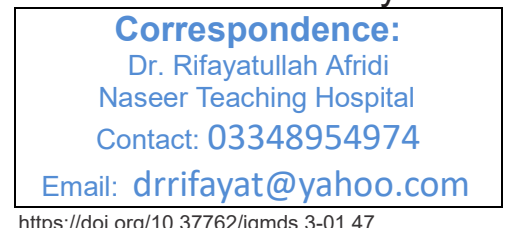
Schinzel. Features include postaxial polydactyly, hallux duplication, macrocephaly, and absence of the corpus callosum, usually with severe developmental delay. Although patients usually share the core features, many other associated abnormalities have also been described. Initially thought to be an autosomal dominant condition, subsequent reports of consanguinity, as well as of affected ACS sibs born to unaffected parents, have suggested autosomal recessive inheritance. The similarity between ACS and Greig cephalopolysyndactyly syndrome (GCPS) has been highlighted by several authors ${ }^{1}$. The inheritance is autosomal recessive based on the reports of recurrences in families and parental consanguinity. The gene responsible for this disease has not yet been identified, although Pfeiffer et al suggested that the gene for ACS may be situated on chromosome 12p, mirror duplication of nearly the entire short arm of chromosome 12 was discovered in a child suffering from ACS ${ }^{2}$. According to another study a novel in-frame deletion KIF7 mutation (p.218-221del) was detected. This is the first deletion mutation in KIF7 described in ACLS and is predicted to disrupt the KIF7 protein within the kinesin motor domain. Also present, in addition to the homozygous KIF7 mutation, were loss of function variants in known ciliopathy genes; AHI1 (p.R830W), BBS2 (p.N70S) and RR.S4 (n M $472 \backslash /{ }^{3}{ }^{3} \mathrm{~W} / \mathrm{e}$ are rennrtinn a race of arrnrallosal syndrome in a 6 month old Pakistani infant boy with superimposed pneumonia and measles.

\section{CASE REPORT}

A 6-month-old infant child weighting $6.5 \mathrm{~kg}$ was admitted for intermittent on and off fever for the last 8 months, episodes of recurrent fits, vomiting, difficulty to breath and complaints of physical and mental developmental delay. The parents of the child were first cousins to each other and they had no other child with such illness. There was a significant developmental delay as the baby could not hold his neck in prone position by extending his arms, he did not usually respond to noises and the baby was reluctant to smile. On examination, the child was unable to support his back and could not sit . He was unable to roll over, he could pick and take food in his hand but could not take it to his mouth. His vocalization and mumbling was not as much as 
compared to a 6 month old child, the facial expressions and smile was not present for food at site and the baby was irritable and cry was high pitched mourning type. Further examination showed wide open anterior fontanelle (figure 1), frontal bossing, depressed nasal bridge, flat occiput ,micrognathism (figure 2), polysyndactyly in both feet (figure 3) and polydactyl finger amputated surgical scars on both hands (figure 4). On further examining the mouth there was high arched palate(figure 5), and ankyloglossia (fused tongue) and koplicks spot could be seen. On examining the chest bilateral crepitation were herd on most of the chest and respiratory rate was more than 65 per minute. Two days later the patient developed maculopapular rash, which was on the face back of ears, and extended down (figure 4) on chest xray multiple small opacities were seen in the right upper and middle lobe and patchy opacity in the lower lobe suggestive of pneumonia (figure 6). CT scan brain showed prominent ventricles, brain atrophy and agenesis of corpus callosum (figure 7) .For further evaluation CSF was taken which was normal without any abnormalities (clear color, total cell count of $5 / \mathrm{cmm}$ in which neutrophils $60 \%$ lymphocytes $40 \%$ was seen CSF sugar was $56 \mathrm{mg} / \mathrm{dl}$ total protein $16.40 \mathrm{mg} / \mathrm{dl}$ with no microorganism seen by staining .Complete blood count raveled $\mathrm{Hb} 10.1 \mathrm{~g} / \mathrm{dl}$, TLC 5800/cmm, neutrophils $78 \%$ lymphocytes $22 \%$ ).

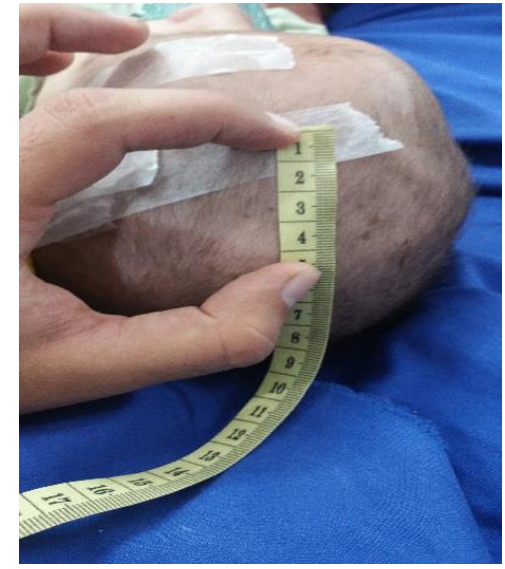

Figure \# 1: Anterior fontanelle of 4 inches in length

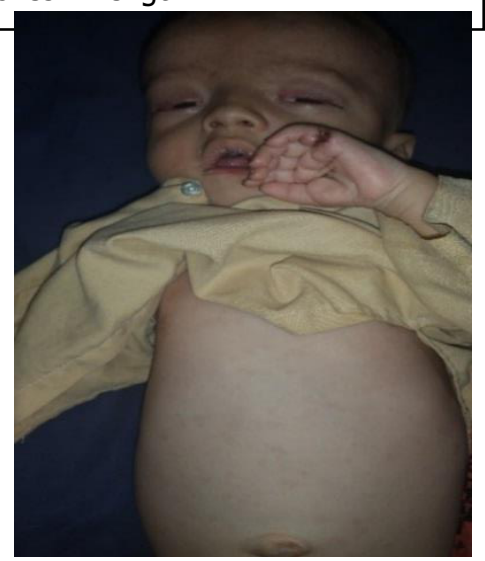

Figure \# 4: Surgical amputated finger scar can be seen on left hand and maculopapular rash on face and abdomen

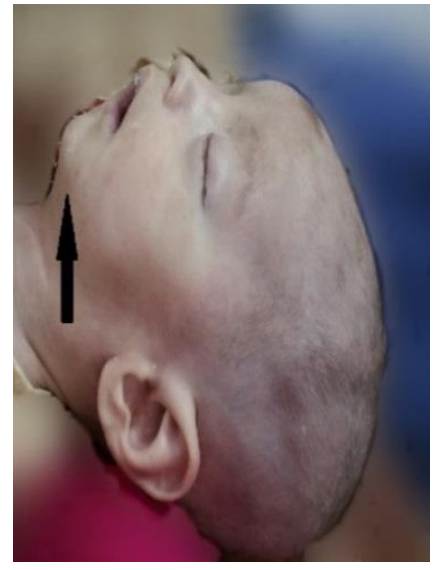

Figure \# 2: Micrognathism

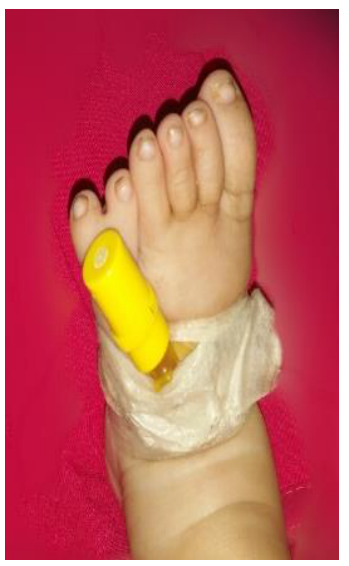

Figure \# 3: Polysyndactyly

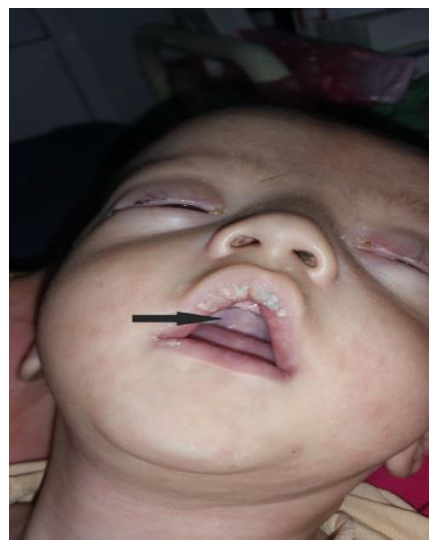

Figure \# 5: High arched palate can be seen the picture 


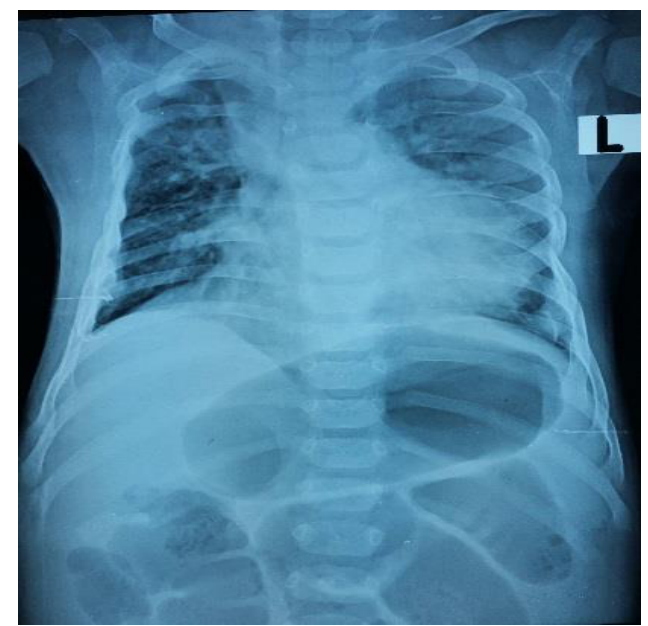

Figure \# 6: Multiple small opacities seen in upper middle and lower lobes of the lunges

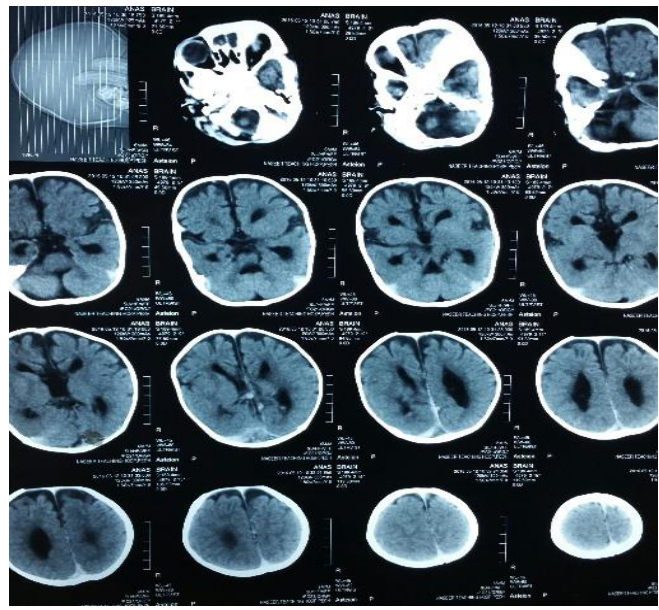

Figure \# 7: CT-brain absent corpus callosum cerebral atrophy and prominent ventricles

A diagnosis of acrocallosal syndrome with measles and superimposed pneumonia was made. Management was started on injectable ceftrixone500 mg which did not respond well and injectable vancomycin was added, vitamin A drops were given for measles, injectable diluted diazepam $0.3 \mathrm{ml}$, and clonazepam: $0.25 \% \mathrm{w} / \mathrm{v}$ drops for treatment of episodes of fits, oxygen supplementation was given after an episode of dyspnea , syrup paracetamol for fever. During the 5 days of admission in the hospital, the patient's condition improved. Multiple well-specialized oriented medical personals were selected for further team management, which included the neurosurgeon, plastic surgeon, orthopedic surgeon physiotherapist and nutritionist for further management and follow up for the concerned problems.

\section{DISCUSSION}

The diagnosis of ACS is based on the criteria laid by Courtens et al. (a) Total or partial absence of the corpus callosum on neuroimaging. (b) Minor craniofacial anomalies (prominent forehead, hypertelorism, short nose with anteverted nostrils and large anterior fontanel. (c) Moderate to severe psychomotor retardation (with hypotonia) and (d)polydactyly. The presence of three of the four criteria is suggestive of the diagnosis of ACLS. ${ }^{4}$ In the presnt case all four features of the criteria are full filled in diagnosing the case as acrocallosal syndrome. Other syndromes similar to ACLS are Joubert syndrome (JS), Hydrolethalus syndrome (HLS), HLS is characterized by hydrocephaly with absent upper midline structures of the brain, micrognathia and polydactyly. Various other features such as cleft lip or palate, club feet, anomalies of the ears, eyes and nose, a keyhole-shaped defect in the occipital bone, abnormal genitalia as well as congenital heart and respiratory organ defects have also been observed in affected individuals ${ }^{5}$ In classic JS is associated with neonatal hypotonia (loss of muscle tone), ataxia, developmental delay, mental retardation, and often neonatal apnea/hyperpnea (irregular breathing) and/or ocular motor apraxia (difficulties in initiating rapid horizontal eye movements- saccades) ${ }^{[6]}$ None of these features alone is diagnostic of JS, however, and in more recent years, it has become obvious that JS is a part of a spectrum of disorders involving vermis hypoplasia and the MTM(molar tooth malformation). Some of these include $\mathrm{COACH}$ (OMIM 216360), referring to characteristic hallmarks of cerebellar vermis hypoplasia, oligophrenia (mental impairment), congenital ataxia, ocular coloboma and hepatic fibrosis ${ }^{7}$. Hydrolethalus is a disorder which shares similar features to ACS such as polydectyly absent corpus callosum prominent forehead hypotonia but the case is 
usually still birth or the new born may survive a few hours after birth .Joubert syndrome is similar to ACS but the patient may have ataxia, cerebellar vermis hypoplasia coloboma etc and to differentiate from ACS if any doubt is present one must do genetic work up to differentiate between the two syndromes (exception for joubert syndrome type 12 which may have the same genotype involvement of kIF7) but as no such facilities were available in our case and the poor socioeconomical status of the patients family for affording such investigations was nearly feeble ,strong clinical features and other investigations including CT-brain supported in the favor of diagnosis acrocallosal syndrome

\section{REFRENCES}

1. Elson E, perveen R, Donnsni D, s wall, et all, , De novo GLI3 MUTATION in acrocallosal syndrome: Broadening the phenotype spectrum of GLI3 defects and overlap with murine models. J Med Genet 2002;804-06.

2. Pfeiffer RA,Legat G, Trautmann U,Acrocalosal syndrome in a child with de novo inverted tendem duplication of 12p11.2-p13.3.Ann genet 1992;35(1):41-6

3. Walsh DM, Shalev SA, Simpson MA et all,Acrocallosal syndrome: identification of a novel kIF7 mutation and evidence for olgogenic inheritance. European journal medicine genetics 2013 jan; 56:39-42.

4. Courtens w, Vamos E, christophec, schinzel A,Acrocallosal syndrome in an Algerian boy born to consanguineous parent:review of the litreture and further delineation of the syndrome.A.M J med genet 1997;69:17-22.

5. Salonen $R$, hava $R$, norio $R$, the hydrolethalus syndrome : delineation of a new lethal malformation syndrome based on 28 patients. clin.genet 1981;19(5):321-330

6. Maria BL, Boltshauser E, palmer SC and Tiran, clinical features and revised diagnostic criteria in joubert syndrome. j.child neurol 1999;14:583-590

7. Gleeson j g, keeler L c, parisi M A, Marsh SE et al, molar tooth sign of midbrain-hindbrain junction: occurrence in multiple distinct syndromes. Am.J.med genet 2004;125-134

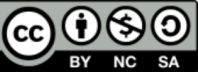

LICENSE: JGMDS publishes its articles under a Creative Commons Attribution Non-Commercial Share-Alike license (CC-BY-NC-SA 4.0). COPYRIGHTS: Authors retain the rights without any restrictions to freely download, print, share and disseminate the article for any lawful purpose. It includes scholarly networks such as Research Gate, Google Scholar, LinkedIn, Academia.edu, Twitter, and other academic or professional networking sites. 